\title{
Tensiones Respecto a la Brecha Digital en la Educación Peruana
}

\author{
CARLOS NARCIZO TARAZONA \\ carlosnarcizo704@gmail.com \\ https://orcid.org/0000-0001-6596-0238 \\ Universidad Nacional Mayor de San Marcos
}

Tensions Regarding the Digital Divideiln Peruvian Education

\begin{abstract}
RESUMEN:
La pandemia por el Covid-19 ha desnudado muchas carencias a nivel mundial, como el aspecto educativo debido a que una de las medidas fue el cambio del escenario presencial al virtual, en las clases. Muchos estudiantes no accedieron, por desconocimiento de las herramientas tecnológicas y de conexión a internet, comprobando la inequidad educativa, principalmente en países latinoamericanos el $55 \%$ han sido afectados, donde seis de cada diez hogares sufren esta necesidad. El presente estudio tiene como objetivo mostrar la desigualdad en la educación digital que está latente pese a los esfuerzos de las políticas educativas implementadas por los gobiernos. La investigación es tipificada como investigación básica, cualitativa y con diseño sistemático; además, se consideró un muestreo de documentos normativos del Minedu y Unesco los cuales abordan el problema de la labor educativa en crisis sanitaria y la sociedad digital, respectivamente. En los resultados encontrados se evidenció que existe la brecha digital por nivel económico, ubicación geográfica: urbano y rural, entre otros. Se concluye que la pandemia ha descubierto mucha desigualdad en la educación virtual y se considera que la tecnología, y conexión a internet son de vital importancia para el cierre de brecha digital en el Perú.
\end{abstract}

\begin{abstract}
:
The Covid-19 pandemic has exposed many deficiencies worldwide, such as the educational aspect because one of the measures was the change from the face-to-face scenario to the virtual one, in classes. Many students did not agree, due to ignorance of the technological tools and internet connection, proving educational inequity, mainly in Latin American countries 55\% have been affected, where six out of ten households suffer from this need. The present study aims to show the inequality in digital education that is latent despite the efforts of educational policies implemented by governments. The research is classified as basic, qualitative research with a systematic design; In addition, a sample of normative documents from Minedu and Unesco was considered, which address the problem of educational work in a health crisis and the digital society, respectively. In the results found, it was evidenced that there is a digital divide by economic level, geographical location: urban and rural, among others. It is concluded that the pandemic has discovered a lot of inequality in virtual education and it is considered that technology and internet connection are of vital importance for closing the digital divide in Peru.
\end{abstract}

\section{Palabras Clave:}

Brecha digital; desigualdad educativa; brecha de acceso a herramientas tecnológicas; brecha de conexión a internet.

\section{KEYWORDS:}

Digital divide; educational inequality; technological tools access gap; Internet connection gap.

Recibido: 23/05/2021 - Aceptado: 29/06/2021 - Publicado: 20/08/2021

\footnotetext{
(C) Los autores. Este artículo es publicado por la Revista peruana de investigación e innovación educativa de la Facultad de Educación, Universidad Nacional Mayor de San Marcos. Este es un artículo de acceso abierto, distribuido bajo los términos de la licencia Creative Commons Atribución 4.0 Internacional (CC BY 4.0) [https://creativecommons.org/licenses/by/4.0/deed.es] que permite el uso, distribución y reproducción en cualquier medio, siempre que la obra original sea debidamente citada de su fuente original.
} 


\section{Introducción}

Una de las dificultades a nivel mundial manifestado en el aspecto educativo, provocado por el Covid-19 es la desventaja del acceso a los recursos tecnológicos. Dicha situación se pone de manifiesto a pesar de que la educación es un derecho y se protege la equidad, pero se ha puesto de manifiesto la falta de igualdad entre los grupos sociales, en el acceso dichos recursos para el desarrollo de las clases remotas; sin embargo, esta pandemia ha traído oportunidades para cambiar esquemas y prepararse para implicancias posteriores (Prince, 2021). Es cierto que la educación y los derechos humanos están íntimamente relacionados debido a que no habría respeto a los derechos humanos si no hay educación pudiéndose considerar que no se puede alcanzar una educación de calidad con muchas diferencias de derecho a esta (Bellver, 2019).

La educación en la emergencia sanitaria se ha desarrollado de manera virtual, mediante clases remotas, usando medios tecnológicos conectados a línea de internet, con la necesidad de las familias para adquirir dichos medios electrónicos, la falta de conocimiento en el uso de las plataformas virtuales y asociado a factores económicos que fueron los motivos para que algunos escolares no pudieran continuar con el normal desarrollo educativo durante los años 2020 y 2021. Sobre ello, países como Espańa, Alemania, Bélgica, China y otros plantearon diversas estrategias para disminuir tal carencia (Martínez, 2020); pero pese a las dificultades la comunicación del maestro entre los estudiantes y padres de familia se hizo por el medio más usado en esta pandemia, mediante la mensajería instantánea del aplicativo WhatsApp de los teléfonos digitales (Montenegro et al., 2020).

En Latinoamérica y países caribeños, la falta de servicios de conectividad digital afecta a un 55\% de los latinoamericanos, donde seis de cada diez hogares sufren y no poseen acceso a internet móvil. Apreciándose ello, de manera mayoritaria, en hogares de bajos recursos y zonas rurales, teniendo en cuenta que la conectividad digital es necesaria para el uso de servicios de comunicación, el acceso a los aparatos tecnológicos en acorde con el avance tecnológico debería ser más accesible (Congreso Latinoamericano de Telecomunicaciones, 2019). También cabe referir que los resultados del aprendizaje y el cumplimiento de los objetivos académicos está relacionado con el acceso a las tecnologías (Montenegro et al., 2020).

A finales del primer trimestre del año 2020, el Perú hace público una medida de emergencia con la emisión del DS. N $\mathrm{N}^{\circ}$ 44-2020-PCM, donde una de las medidas es que los ciudadanos permanezcan en sus casas y donde se restringe la movilidad de estos. A nivel de las familias, los maestros ni los estudiantes estábamos preparados, razón por la cual la comunicación entre maestros y estudiantes no fueron posibles hasta el segundo semestre del año (Presidencia del Consejo de Ministros, 2020). Viendo tal situación el Instituto Nacional de Estadística e Informática (INEI), que hacía una investigación sobre las tecnologías de información y comunicación (TIC), cambio a las entrevistas telefónicas. Dentro de los datos obtenidos, se registró que en el 2019, el 93,9\% de hogares había al menos una TIC; mientras que, en el ańo 2020 se incrementó a 94,3\% con un pequeño crecimiento de 0,4\% (INEI, 2019).

En el Perú se tiene que el 38,4 de la población peruana son adultos mayores de 70 años y viven solos siendo notorio en los departamentos de Puno, Huancavelica y Apurímac; mientras que, de este porcentaje el 61,8\% viven con otras personas y el 38,2\% viven solos. Lo que implica también la inequidad del uso de medios digitales por la edad y por desconocimiento y consideradas como personas vulnerables (Instituto Nacional de Estadistica e Informática, 2018).

Para Cortés (2010) la brecha digital es definida como la distancia social entre los que poseen y no acceso a las TIC. Sabemos que el avance tecnológico es rápido y aparecen nuevas herramientas tecnológicas, aunque ello no modifica esta inequidad debido a que dificulta la adquisición por motivos económicos. Además existe una coincidencia al definir la brecha digital (Martínez, 2020) como la expresión de desigualdad de uso y acceso a las TIC y a esto podemos agregar la falta de competencias de su manejo. De hecho se busca la equidad en el manejo y uso de medios digitales donde todo ser humano debería tener el acceso a internet y tener el dominio de su uso, puesto que permite la comunicación sincrónica y asincrónica con otras personas, 
la falta del acceso de este medio dificulta la educación, el negocio en línea y el emprendimiento online (de Andrés et al., 2020).

Según Yansen y Zukerfeld (2013) se puede señalar que en la sociedad tanto niños como niñas se han ido desarrollando en el mundo de la tecnología desde un principio por juguetes que los padres adquirían para sus hijos. La existencia de juguetes masculinos y femeninos sugería que para un niño había más opciones de compra de un juguete que para las nińas. Ello traía como consecuencia que los niños tenían más curiosidad que las niñas en averiguar ¿qué hay dentro del juguete? ¿cómo funcionan?, otro aspecto resaltante fue el inicio de los videojuegos que tuvo como usuarios frecuentes a los niños. La aparición del internet también marca una diferencia en grupos de chat y la posibilidad de acceder al uso social de la computadora, los adolescentes varones tuvieron un acercamiento a las computadoras de temprana edad, y eso les motivó a ingresar al mundo de la programación. Actualmente hay mujeres desarrollándose en este campo con predominancia a la comunicación, el diseńo, tareas administrativas, manejo de personal y otros. Se precisa que el $24 \%$ de los trabajadores en programación son mujeres mientras que la vinculación de los varones se relaciona por su inmersión por los juegos en la niñez.

Se dice que la educación debe ser igual para todos, pero existen diferencias que la agruparemos en dos dimensiones. Primero, el hogar de origen y que se entiende como la inequidad que se da de generación en generación, se enmarca a la oportunidad generacional o a las restricciones a la educación desde los hogares desamparados, lo que no sucede con los hogares de estratos superiores. Segundo, el sistema educativo genera una educación mercantilista, que urge la acreditación de la educación para el acceso a las condiciones básicas de bienestar social y la calidad educativa del país. En ese sentido va quedando claro que la inequidad educativa tiene como base la desigualdad social por lo que el Estado debe promover políticas sociales direccionadas a superar la dimensión hogar (Menese, 2020). La inequidad en la educación también está asociada a la pobreza, es decir, hay una diferencia entre la educación en zonas urbanas y las rurales, donde el Estado muy poco invierte debiéndose priorizar la educación como un derecho social (Junca, 2017).

La pandemia a causa del Covid-19 ha traído muchas dificultades y un escenario que nos permite distinguir la oportunidad de dar un gran paso a la educación digital y estar preparados para una situación como esta. Asimismo, es momento que los gobierno se responsabilicen de ella, la consideren como un gran reto y propongan como perspectiva jurídica a la educación digital como un derecho y evitando de esta manera la inequidad en la educación digital (Cotino, 2021). Desde hace mucho tiempo existe el analfabetismo de la lectoescritura, hoy continua, pero como un analfabetismo digital, latente en esta inequidad de la educación. Una de las modalidades de la educación es la educación a distancia, pero ahora se debe dar énfasis en la educación a distancia virtual, para disminuir la brecha digital y la exclusión social (Martínez, 2018).

La educación virtual es una novedosa experiencia pedagógica para maestros y estudiantes, lo cual esto involucra responsabilidad, compromiso e iniciativa; aunque demanda a los maestros un mayor tiempo para organizar sus materiales; pero luego la interacción con el estudiante en tiempo real es muy favorable, debido a que se puede notar claramente el estado de ánimo tanto del maestro como del estudiante durante el desarrollo de la clase (Esteban et al., 2020).

En esta era de la tecnología digital, la educación debe reorganizar su estructura educativa en base al uso de plataformas digitales donde el hombre requiere del almacenamiento masivo de datos (Big Data), la creación de máquinas, herramientas y robots que imiten al hombre en el trabajo y en el campo educativo, haciendo uso de la inteligencia artificial (IA) para dar nuevas perspectivas de futuro en el campo educativo (Bonami et al., 2020).

Adicionalmente, el avance de la tecnología trae consecuencias negativas para el hombre como mencionan Toyama y Rodríguez (2019) al referirse que una máquina reemplaza al hombre en el mundo laboral y se cuestionan mencionando donde quedarían los derechos laborales de la persona que ha demostrado un desenvolvimiento óptimo en su puesto de trabajo. En este caso el Big Data y la inteligencia artificial estarían jugando en contra del bienestar del trabajador y su familia, pero cuando la empresa tiene un puesto de trabajo 
y hay varios postulantes, se puede buscar información masiva del comportamiento del trabajador mediante redes sociales, plataformas digitales, LinkedIn e Instagram, lo cual puede ser beneficioso o perjudicial para el postulante, debido a que puede encontrar datos en su contra o a favor que el empleador puede usarlo para tomar decisiones finales.

Por el lado del docente, se asume el reto de la realización de un trabajo remoto ante la pandemia del Covid-19, debido a que de manera imprevista, se ha tenido que desenvolver en las clases virtuales, a pesar de la falta de desarrollo de competencias digitales, pero con voluntad y anhelo para optimizar la calidad de la educación haciendo un esfuerzo para adquirir tales competencias con sus propios recursos (Velásquez, 2020). Ante el contexto actual, el docente tiene un gran desafío, pese a que algunos carecen de habilidades y manejo de uso de herramientas digitales, pero el reto está en integrar las TIC a los procesos formativos de los estudiantes de pedagogía. Estos últimos deben de adquirir competencias ya sea en aspectos técnicos, formativos y metodológicos de las nuevas herramientas para que luego puedan adquirir autonomía y responsabilidad en su aprendizaje (Sandoval, 2020).

Los nacidos después de los años 80 son conocidos como nativos digitales, aunque se sigue observando claramente la inequidad digital debido a aspectos económicos, pues no pueden acceder a los aparatos tecnológicos modernos. Esto determina que sean potenciales proactivos de dominio frente a los pasivos de dominio tecnológico, denominados como falsos nativos digitales. La digitalización de la educación es un reto que requiere un cambio en el sistema educativo, la construcción de un nuevo paradigma educativo en entornos virtuales para el desarrollo de una educación integral y competente para el desarrollo de la sociedad (Granado, 2019). Es difícil referirnos a los nativos digitales, debido a que a pesar de haber nacido en este mundo globalizado y con habilidades digitales altas, puede haber otros que no tengan las mismas capacidades por diferentes variables, como vivir en regiones urbanas, regiones rurales, naciones y otros (Gallardo, 2013).

Hemos mencionado que el Covid-19 suponía una oportunidad para dar un paso adelante en la educación digital y dejar atrás la educación tradicional en la pizarra y cuatro paredes, pero el confinamiento obligó al desarrollo de la educación en casa. Ello se visibilizó en la educación en familia manifestado en la gama de dificultades generando una brecha muy alta entre los que no poseen acceso a herramientas digitales y conectividad de internet. Decimos entonces que solo fue un complemento, pero no se lograron cumplir las metas educativas, ni el derecho a la educación; mientras que, el aprendizaje en casa ha sido resuelto por las familias de acuerdo a sus posibilidades económicas y sociales, pero en lo sucesivo, la educación digital podría ser un salvavidas para casos similares de confinamiento social (Cotino, 2021). Pero también Gómez (2021) menciona que por ejemplo, a pesar de las grandes dificultades que se tuvo en la estrategia Aprendo en casa, se ha podido lograr un desarrollo en caso de la competencia de comunicación oral y que un factor importante ha sido el compromiso de los padres de familia en apoyar a sus hijos expresado en guiarlos en el proceso de lectura en sus casas.

En el siglo XXI, donde el avance de la tecnología va muy rápido, el acceso a los medios digitales y conexión a internet se está convirtiendo en un derecho social, lo que nos hace reflexionar que dicho avance tecnológico debe ser más inclusivo que exclusivo en los diversos sectores de la sociedad. Acotando que existen personas que viven con un sueldo que no alcanza ni para la canasta familiar, lo que es limitante para la adquisición de medios tecnológicos de última generación (Cisneros, 2017). La brecha digital en Latinoamérica trae consigo la poca investigación, producción científica y proyectos tecnológicos en robótica e industria, la necesidad que las empresas y el gobierno se centren en la triangulación, comunicaciones mediante los móviles digitales, lo cyber-físico y la hiper-conectividad, es decir, la relación cliente-industria-gobierno para el desarrollo y la competitividad (Corzo y Alvarez, 2021).

El uso tecnológico en los adultos mayores es de gran importancia tanto de quienes viven solos o acompańados por familiares debido a que mantiene su independencia al utilizar los medios tecnológicos para realizar sus compras o hacer sus pagos de servicios, pedir citas por teléfono y otros. De hecho, eso impacta de manera positiva en el bienestar del adulto mayor, tanto socialmente y económico; sin embargo, no 
pueden hacerlo los que viven en lugares que carecen de servicios de telefonía, internet y aparatos tecnológicos. También reconocer que es importante la inclusión del adulto mayor y las TIC para mejorar su calidad de vida (Aguilar y Chiang, 2020).

Antes de la pandemia muchos colegios públicos, privados en el Perú y hasta el mismo gobierno no daban mucha importancia la educación virtual, más bien daban prioridad al aprendizaje de manera presencial, pero es hora de dar saltos hacia una mixtura de la educación presencial y digital para lograr un aprendizaje de calidad, donde la educación no sea un privilegio sino más bien de equidad (Barón y Caicedo, 2021). La igualdad de oportunidades en el Perú está en riesgo debido a que la brecha social de inequidad generado por la pandemia de Covid-19 en el año 2020, ha puesto de manifiesto la diferencia de oportunidades en el uso de herramientas tecnológicas, para desarrollar sus clases de la estrategia Aprendo en casa, por la ubicación geográfica, recursos económicos, experiencia en el manejo digital y otros (Gómez y Escobar, 2021).

Ruiz (2017) refiere en una investigación que el aprendizaje en entorno virtual favorece el desarrollo de competencias mediante el uso de las TIC en el proceso de enseñanza y aprendizaje integral de los maestros. Además, se hace necesario difundir las buenas prácticas docentes mediante reconocimientos por parte del ente educativo y con los materiales de participación de los maestros. Adicionalmente, propone organizar un repositorio para una formación activa para los docentes mediante infografías, videos y otros materiales TIC. Precisamos que para complementar la propuesta faltaría mencionar la formación de comunidades virtuales de aprendizajes con otras instituciones educativas para compartir experiencias de tal manera que en la educación sea de uso a la virtualidad en el intercambio de competencias entre docentes y estudiantes para reducir la brecha digital.

\section{Método}

El propósito de la investigación es describir la inequidad de la educación digital en la sociedad actual a partir de la inequidad social que ha exhibido la pandemia. Esto ha evidenciado que algunos estudiantes no accedan a sus clases remotas a tiempo por diversas razones, y mientras que otros, sí pudieron enfrentar las clases virtuales. Esto último, nos conlleva a la indagación de las causas de esta desigualdad y saber si los representantes de los gobiernos contribuyen a superar esta deficiencia, hasta el momento la educación diferenciada muestra un peligro para una educación con derecho social e inclusiva.

Para el desarrollo de la investigación se utilizará el enfoque cualitativo como lo plantea Valdez (2018) al precisar que este posee un conjunto de caminos diversos con un abordaje particular. Además, presenta procesos de desarrollo de la información para la comprensión de un problema en tres niveles: el estudio de la realidad social sin intentar alterarla, manteniendo toda su complejidad; descubrir de manera crítica la realidad para incidir en la práctica diaria y, lo que ello implica, el discernimiento, la comprensión y la transformación de la construcción de una nueva sociedad.

La investigación es de tipo básica (Sánchez y Reyes, 2015) pues se orienta a explorar informaciones recientes con la intención de recabar información de la realidad para perfeccionar el conocimiento científico para el descubrimiento o llevar al logro de conseguir nuevas conocimientos o teorías, siendo el principal objetivo es la generación de información para potenciar el conocimiento científico. En tal sentido, se analizaron los documentos provenientes del Ministerio de Educación los cuales establecen la normativa y las orientaciones para el servicio educativo en el contexto de la emergencia sanitaria. Por otro lado, se tuvo en cuenta el documento proveniente de UNESCO el cual aborda la complejidad de la sociedad digital a partir de las brechas y retos existentes.

\section{Resultados}

Los resultados del análisis documental incluyen la Resolución Viceministerial RVM No093-2020-MINEDU titulada Orientaciones pedagógicas para el servicio educativo de Educación Básica durante el año 2020 en el 
marco de la emergencia sanitaria por el Coronavirus COVID-19. En este primer documento normativo del Minedu se pueden resaltar los siguientes aspectos.

\section{a. Las actividades}

Según el documento analizado, las actividades se desarrollarán a través de la estrategia Aprendo en casa, que propone una educación remota desde el domicilio del estudiante, con un acceso libre que propone experiencias de aprendizaje propuestas en el currículo nacional en base a la disponibilidad del estudiante según sus posibilidades. Dentro de ellas, se consideran los medios de acceso a ello, como el uso de la plataforma educativa virtual de Aprendo en casa. Esta contiene orientaciones para los padres de familia, estudiantes y maestros, así como también actividades para los diferentes niveles y modalidades. En el caso del nivel inicial se requiere mayor apoyo del padre de familia en el nivel primaria las actividades hacen que el estudiante conozca su entorno y promueva su bienestar, para fortalecer el desarrollo del aprendizaje; mientras que, en nivel secundaria los estudiantes pueden trabajar con cierto grado de autonomía, fortaleciendo sus competencias para una convivencia en democracia, el buen ejercicio de la ciudadanía y sobre todo resolver situaciones de su vida cotidiana.

En el caso del medio televisivo, las experiencias de aprendizaje son transmitidas a través de la televisión nacional del Perú (TVPERU) y otros canales privados, las programaciones son publicadas vía Facebook del Ministerio de Educación, plataforma web, los estudiantes son orientados por el conductor (a), dando pequeńos espacios para la reflexión del estudiante. Se suma a ello, la transmisión por radio, la programación es publicada por los medios mencionados anteriormente, en este caso las experiencias de aprendizajes son transmitidas por todas las radios a nivel nacional. Ello es programado con una diferencia de tiempos: para el nivel inicial en un lapso de 15 minutos; mientras que, para primaria y secundaria son de 30 minutos. Se señala que la ventaja de este medio es que los estudiantes lo pueden escuchar en diferentes lenguas originarias y como solo se puede oír, esto permitiría desarrollar la imaginación, creatividad y reflexión para desarrollar y fortalecer sus aprendizajes.

\section{b. Las orientaciones para la labor de los directivos}

Luego de la revisión de la normativa realizada se podría considerar que bajo la modalidad de la educación a distancia el líder pedagógico, responsable de la institución educativa, debe generar espacios de concertación con los docentes de todas las áreas para revisar las experiencias de aprendizajes planteadas por el Ministerio de Educación. Esto último con la finalidad de que puedan hacer reajustes en la planificación curricular considerando las necesidades de los estudiantes, informar a los padres de familia, las expectativas establecidas por acuerdo en la institución educativa, para generar compromisos en la comunidad educativa; proporcionar orientaciones a los docentes con apoyo de instituciones aliadas para generar un clima de acogida y soporte emocional para los estudiantes y sus familias.

Además, propiciar la comunicación del docente con sus estudiantes y familiares para confirmar que se logra el uso de recursos que brinda el Ministerio de Educación en la estrategia Aprendo en casa. Asimismo, generar la búsqueda de soluciones en equipo a través del trabajo colegiado, incluir aspectos significativos que incluyan la orientación de los docentes sobre el monitoreo hacia los estudiantes en el uso correcto de las estrategias planteadas por la estrategia, el uso del portafolio y e-portafolio como fuente de evidencia de aprendizajes de los estudiantes.

\section{c. Las orientaciones pedagógicas del docente}

Seguido de la revisión del documento normativo se debe tomar en consideración que el docente de manera personal deberá revisar los materiales dotados por el Ministerio de Educación en la estrategia por sus tres formas de comunicación: plataforma web, radio y televisión. El docente debe estar en contacto permanente con el estudiante, sus familias y organizar momentos de acogida con ellos, dando a conocer la crisis sanitaria y motivando el cuidado de la salud física y mental. Para la planificación de las experiencias de aprendizaje 
contextualizado y en función a las necesidades del estudiante, el maestro debe prever los insumos necesarios como la revisión de los recursos de la estrategia señalada, identificar el medio o aplicativos disponibles.

Además, debe de considerar si cuenta con conectividad disponible para la comunicación, el monitoreo respectivo, la presentación de sus evidencias de aprendizaje y su retroalimentación respectiva, fomentar el uso del portafolio y e-portafolio; considerar también la situación del aislamiento social para que la familia pueda contribuir en la formación educativa de los hijos con actividades propuestas por el maestro, actividades de diálogo, reflexión familiar e intergeneracional de saberes ancestrales de su cultura, costumbres, relatos y tradiciones para fortalecer la identidad cultural.

\section{d. El Monitoreo, Evaluación y Retroalimentación al Proceso de Aprendizaje}

Según el análisis del documento, durante la modalidad a distancia los docentes deben identificar los medios adecuados y organizar el monitoreo respectivo a sus estudiantes con el fin de verificar el uso adecuado y el acceso de la estrategia Aprendo en casa. Ello se debe de registrar a través de las evidencias de aprendizaje en el portafolio físico o virtual de toda la colección de las producciones realizadas por el estudiante. Esto último con la finalidad de realizar la evaluación respectiva y considerando a la evaluación como el recojo de información y desde un punto de vista formativa con la finalidad de proporcionar el apoyo al estudiante, lograr su permanencia, evitar el estrés y el abandono escolar. Luego de ello, realizar la retroalimentación formativa que se hará efectiva devolviendo al estudiante, la evidencia de sus desempeńos. En este momento se describen sus logros de aprendizaje que se relacionan con las metas de aprendizaje propuestas en el currículo nacional. Se incorpora también que si el estudiante, por alguna razón está en un nivel de proceso, el maestro realizará el acompañamiento a los estudiantes de manera remota en coordinación con el padre de familia para proporcionarle apoyo.

\section{Resultados según análisis documental normativo de la Resolución Ministerial N400-2020-MINEDU}

La revisión del documento emitido por el Ministerio de Educación, donde aprueba los lineamientos de distribución de tabletas para los estudiantes de colegios de zonas rurales de extrema pobreza con la finalidad de continuar las clases remotas. De hecho, no se pretende lograr el desarrollo de todas las competencias planteadas, pero como menciona en el documento el Ministerio de Educación se trata de superar de la mejor manera esta desigualdad educativa. Esto con la finalidad de seguir consolidando las competencias restantes y enfatizando las competencias educativas contextualizadas esta situación educativa y como una forma de disminuir la brecha digital.

\section{a. La distribución de las tabletas y sus complementos.}

La normativa vigente analizada indica que la distribución de las tabletas será ejecutada por el personal de la Dirección de Gestión de Recursos Educativos del Minedu (Digere) posee dentro de sus funciones: la planificación, la programación del presupuesto para la distribución de los materiales educativos a nivel nacional y luego monitorear el estricto cumplimiento de la normativa con el apoyo de los gobiernos regionales. Además, brindará asistencia técnica a la Dirección Regional de Educación (DRE), Gerencia Regional de Educación (GRE) y Unidad Ejecutora Local (UGEL), para planificar y gestionar el proceso de distribución física y se dé cumplimiento a las normas educativas pertinentes, a la DRE y ellas a la UGEL, el responsable de cada Unidad Ejecutora realiza las programaciones para realizar la entrega a los líderes pedagógicos de las instituciones educativas.

\section{b. La recepción de las tabletas en las instituciones educativas públicas y entrega a los beneficiarios}

Según la revisión del documento indica que el director de la institución educativa y la comisión de Gestión de Recursos Educativos de cada institución recibirán las tabletas previo acuerdo de fecha considerada con los 
responsables de la entrega de la comisión del Ministerio de Educación y UGEL. Para ello, se considerará la cantidad, las características de fabricación como indica en el reporte, serie y demás objetos que complementan su funcionamiento ejecutando la revisión presencial de cada tableta; si se nota algún deterioro o fallo del sistema u otro defecto ya sea en la tableta o sus complementos esta no será recibido, y pactaran una fecha para la entrega complementaria. Igualmente, una vez la entrega sea dada como conforme, el encargado de la recepción procederá a dar la conformidad. Luego, el director y la comisión tendrá un plazo de cinco días para realizar la entrega a los beneficiarios para que luego la UGEL, DRE o GRE realice el registro en el Sistema Integrado de Gestión Administrativa-Módulo o Patrimonio (SIGA-MP) de lo entregado.

Luego, la misma comisión de recepción harán entrega a los padres de estudiantes beneficiarios. Para el caso de los docentes, la entrega será de manera personal, verificando por ambas partes, la totalidad de sus complementos, comprobando el buen estado y funcionamiento. Los estudiantes beneficiarios son de: $4^{\circ}$ grado al $6^{\circ}$ grado en el nivel primaria, y de $1^{\circ}$ al $5^{\circ}$ grado del nivel secundaria. Igualmente, si hubiera sobrantes de tabletas por motivo de retiro o traslado de estudiantes, se entregará a estudiantes que no fueron prioridad para dicho beneficio y si aún existen sobrantes el director informará a la UGEL correspondiente para su devolución. De la misma manera, la comisión deberá informar que las tabletas se entregan en condición de préstamo, por lo que el padre de familia y maestro deberán devolverlos al finalizar el año académico, lo que implica el cuidado respectivo como indica en la guía o instructivo sobre el cuidado de esta.

\section{c. Los contenidos, recursos y aplicativos instalados en las tabletas}

Según el análisis del marco normativo, las tabletas son periféricos o dispositivos digitales portátiles donde los usuarios ingresan información a través de la pantalla táctil, siendo dispositivos con menor consumo de energía eléctrica. Dichas tabletas están configuradas con algunos servicios digitales instalados que dependen del nivel educativo de los estudiantes. Dichos recursos fueron aprobados por las direcciones pedagógicas del Minedu, donde cada beneficiario, sean estudiantes o maestros, pueden acceder a diversos servicios digitales, accesible de inmediato, para los que disponen de conexión a internet, y para zonas sin conectividad. Y, cuenta con gestor de contenidos digitales y aplicaciones instaladas que ayudan el desarrollo de competencias, las herramientas electrónicas tienen filtro de contenidos para evitar el inadecuado uso y el acceso a contenidos que no ayuden al desarrollo educativo del estudiante. Para las zonas que no disponen de energía eléctrica algunas tabletas disponen de cargadores solares portátiles, y cuentan con garantía de fábrica de un año, mientras que las tabletas también poseen garantía de fábrica de un año y medio.

\section{Resultados según el documento de la Unesco Sociedad digital: brechas y retos para la inclusión digital en América Latina y el Caribe}

\section{a. Las tendencias en la adopción de Internet y la brecha de demanda}

En dicho documento se hace referencia a la desigualdad en la tendencia de la adopción de internet y la brecha de la demanda, donde las tendencias más destacables que se mencionan son: el crecimiento del acceso a la telefonía móvil, que en al año 2008 alcanzó un máximo de 18.7 líneas de cada 100 habitantes. Se podría decir que ha aumentado la adquisición de telefonía móvil, pero ha sido un crecimiento lento en la población conectada a internet. Y, hay personas que poseen dispositivos móviles básicos que son usados solo para llamadas telefónicas y sin acceso a internet.

A medida que se expande la banda ancha móvil 4G, se nota un aumento de brecha en la demanda, en los países de bajos ingresos como Honduras, Bolivia, Perú y otros. El nivel de adopción de internet apenas logra alcanzar el $50 \%$ en el quintil de mayores ingresos. Ello, representa un reto para resolver estas diferencias. Por otro lado, las autoridades gubernamentales, con el apoyo de las empresas privadas y organizaciones sociales, deberían organizar proyectos y planteamientos de políticas de cierre de brecha digital. Se enfatiza que, en la mayoría de los casos, la barrera de adopción a internet se ocasiona por el excesivo costo que genera que los jefes de hogares no adopten una línea de internet. Esto último se vio reflejado por falta de interés, según la encuesta de hogares. 


\section{b. Las características de los no conectados}

Según el análisis documental los no conectados son personas que a nivel mundial tienen restricciones en el acceso a la línea de internet. Asimismo, la demanda del uso individual de internet en los hogares depende de diversos factores, como: el aspecto económico ya mencionado anteriormente y donde el jefe de hogar no puede cubrir los gastos de la línea de internet, por el alto costo que no puede solventar. Otra característica es el nivel educativo, donde en caso de las personas con primaria incompleta en Honduras solo el 5\% tiene línea de internet; mientras que, en los países de Perú, Ecuador, Colombia y Bolivia menos del $10 \%$.

Se enfatiza que las personas con el nivel superior, ya sea completa e incompleta, de los países como Bolivia, Brasil, Colombia, Ecuador superan el 80\% en el acceso a internet, ello, nos indica que el grado de instrucción también influye en el acceso a internet. Por otro lado, la ubicación geográfica es otra característica notoria, pues las personas que viven en zonas rurales de los países como Bolivia, registran que el $4 \%$ tiene acceso a internet. En ese mismo sentido se refiere que Honduras registra el 5\%, Paraguay $9 \%$ y solo en Uruguay el $48 \%$ de los habitantes de ruralidad tiene acceso a internet. En contraste a los que viven en zonas urbanas, donde es superior en cada país. Un ejemplo de ello es Bolivia que registra el 20\%, Honduras el 26\%, Paraguay el $32 \%$ de los que viven en zonas urbanas tienen acceso a internet, mientras que en Perú, Brasil y Uruguay superan el 50\%. El panorama anterior deja claro que lo no conectados son los habitantes de zonas rurales en mayor porcentaje.

Otra característica por indicar es el familiar que está compuesto por familias que poseen miembros en edad escolar, más conectadas a internet residencial en comparación con la que no tienen niños en edad escolar se diferencian en dos \% en Bolivia, es decir, del 14\% sin niños en edad escolar en oposición al 16\% de familias con edad escolar. En el caso de Brasil hay una diferencia mayor del 13\%, es decir de $53 \%$ a $66 \%$ y la brecha de género es una característica notable. Esto se visibiliza en Bolivia donde el 38\% de las mujeres usan internet y lo hombres en un $45 \%$ con una diferencia del siete $\%$ y en Perú, las mujeres $47 \%$ y hombres el $54 \%$ existiendo una diferencia del siete \%. En Uruguay, Colombia y Paraguay hay una igualdad con el 71\%, 58\% y 59\% cada uno, mientras que en otros países como Brasil el $58 \%$ de los hombres tienen acceso a internet mientras que el $59 \%$ de las mujeres tienen acceso. En este caso, las mujeres superan en uno \% a los hombres y de los factores mencionados, existen relaciones entre educación y conectividad de línea de internet con el ingreso económico en el hogar de la familia.

De igual manera, la brecha digital de edad y género que se expresa en que los nińos y los adultos mayores no disponen de aparatos móviles conectados a internet y las mujeres de edad escolar, adolescentes y adultas de zonas rurales no tienen acceso a herramientas tecnológicas y menos aún a conectividad de internet de banda ancha. Esto último, conlleva un reto de gran importancia sobre todo en los países de ingresos bajos como Bolivia, Perú, Honduras y otros como Brasil, Uruguay, por señalar algunos.

\section{c. Las recomendaciones}

De acuerdo con el documento analizado, la situación educativa es crítica pues la comunidad educativa en su conjunto hace esfuerzos en coordinación con los padres de familia y el apoyo de empresas privadas para la adquisición de herramientas tecnológicas para ser usadas por los estudiantes. Las recomendaciones según Unesco para reducir las brechas digitales son varias, siendo las más resaltantes: conectar a las escuelas debido a que poseen un papel fundamental en la educación y se ve la necesidad de realizar el equipamiento con herramientas tecnológicas y líneas de internet de banda ancha. Esto con la finalidad de brindar una educación de calidad acorde con el cambio a la virtualidad, realizar la alfabetización digital a padres y maestros, para el apoyo educativo en la familia y maestros para desarrollar actividades haciendo uso de las nuevas tecnologías.

La virtualidad en la escuela ayudará en la preparación de los niños para el desarrollo tecnológico y el empleo del futuro. También, propone que cada gobierno promueva servicios de línea de banda ancha para los no conectados, en tres criterios fundamentales: a los adultos mayores debido a que posee muchas dificultades en competencia digital y barreras de acceso, siendo un desafío para el gobierno. El segundo criterio es promover 
la interculturalidad y fomentar la conectividad entre los hablantes de diferentes lenguas originarias debido a que son de gran importancia para preservar el patrimonio cultural de cada país. Y, el tercer criterio, con una sugerencia de la Unesco, es abordar la problemática de discapacidad debido a que estudios manifiestan que también se presentan barreras de conectividad en dichos ciudadanos.

Profundizando en el tercer criterio se refiere como una prioridad relacionada a los servicios públicos para personas con discapacidad visual y auditiva. Otra recomendación es la implementación de programas de transferencia condicionada que consistiría en invertir en subsidios de conectividad focalizado en hogares de bajos ingresos con condición de asistencia escolar. Y, esto con un efecto de ayuda también para los adultos mayores y personas con discapacidad, de esta manera se estaría cumpliendo con la meta de la inclusión social, es el reto de los gobiernos para generar políticas de inclusión digital.

\section{Discusión}

Durante el análisis de la documentación se identificó que el Ministerio de Educación aprobó varias normativas mediante la estrategia de cierre de brecha digital. La primera normativa propone sobre las Orientaciones pedagógicas para el servicio educativo de la educación básica durante el año 2020 en el marco de la emergencia sanitaria por el Coronavirus COVID-19. Prioritariamente se tiene las orientaciones del desarrollo de las actividades durante el confinamiento social, mediante la estrategia educativa Aprendo en casa. Dicha estrategia básicamente se desarrolla por tres medios, la plataforma web aprendo en casa, la transmisión de radio y televisión. Las estrategias presentadas son para todas las modalidades y niveles de la educación básica, siendo la radio un medio más usado por los estudiantes de zonas rurales debido a que la transmisión fue por diversas lenguas originarias.

Además, las orientaciones en la labor que desempeñan los líderes pedagógicos son fundamentales para generar espacios de concertación entre la comunidad educativa mediante el trabajo colegiado. Ello con la finalidad de determinar acciones y estrategias para el buen desarrollo virtual de aprendizaje, generando confianza y compromiso hacia los padres de familia para el apoyo en el trabajo remoto y la organización de su portafolio y e-portafolio de sus hijos. En ello, el docente desempeña un papel fundamental pues debe revisar los materiales dotados por el Ministerio de educación en la estrategia web Aprendo en casa.

Posteriormente, elaborarán experiencias de aprendizaje, según las condiciones del estudiante en el uso de una de las estrategias presentadas para las clases remotas. Dichas experiencias son elaboradas considerando el contexto real del estudiante para desarrollar con integración de la familia y sus saberes ancestrales, costumbres y tradiciones para fortalecer su identidad. Todas las actividades son monitoreadas y retroalimentadas por el docente por los medios de comunicación generando apoyo, confianza y fomentando el uso del portafolio y e-portafolio, registrando con ello, los logros de aprendizaje de cada estudiante (Ministerio de Educación, 2020b).

Los estudiantes de zonas rurales poseen dificultades para desarrollar sus clases remotas y aunque existan formas de mejorar aprendizajes a través de la radio, la televisión y la plataforma web que favorezcan su aprendizaje, no son suficientes. Debido a esas carencias el Ministerio de Educación decide realizar la entrega de herramientas electrónicas presentando una segunda normativa donde señala las indicaciones y lineamientos para la gestión de tabletas en las instituciones públicas. Preferentemente, indica que la distribución de las tabletas será ejecutada por el director de la DIGER, previa coordinación con los gobiernos regionales y unidades ejecutoras.

A su vez, estas últimas coordinarán con los líderes pedagógicos de cada institución educativa y en coordinación con la comisión de recursos educativos y mantenimiento de infraestructura de cada institución educativa ejecutarán la distribución a los beneficiarios, estudiantes del nivel primaria con excepción de $1^{\circ} \mathrm{y}$ $2^{\circ}$ grado, y estudiantes del $1^{\circ}$ al $5^{\circ}$ de secundaria. Estos últimos considerados en los quintiles uno y dos de pobreza y maestros de las áreas priorizadas en el nivel secundaria como matemática, comunicación, ciencia y ambiente, desarrollo personal ciudadanía y cívica y ciencias sociales. 
Las tabletas tienen diversas aplicaciones seleccionadas y contenidos virtuales como videos, videos en lenguaje de seńas, y contenidos para estudiantes con necesidades especiales, aplicaciones para matemáticas, ciencias, informática y otros. Y para las zonas altoandinas y amazónicas donde no poseen internet vienen con un chip de datos para navegar en la web. La tableta tiene un gestor de contenidos con aplicaciones en lenguas originarias para la educación intercultural bilingüe como la comunicación en lengua nativas (ColeNa), aymara, mamaru entre otras, con esto el Ministerio de Educación garantiza la continuidad de la educación en marco de la estrategia cierre de brecha digital (Ministerio de Educación, 2020a).

La presente investigación tiene coincidencias con los resultados de otros estudios que resaltan la necesidad de dar cambios para una educación con derecho social, donde las tendencias de adopción de internet y la brecha de la demanda ha mejorado en estos últimos años. Dicha mejora, se manifiesta en cuanto a la adquisición de la telefonía móvil, pero el crecimiento es lento en la adquisición de conectividad a internet, en los países con bajos ingresos económicos como: Honduras, Bolivia, Perú, son más notorios debido a que apenas logran alcanzar el $50 \%$ en el quintil de mayores ingresos. En los países mencionados los jefes de hogares no logran acceder a una línea de internet, mayormente por el aspecto económico. Y, el aspecto educativo es otra causal, en Honduras solo 5\% de las personas con primaria incompleta tienen acceso a internet; en Bolivia, Colombia, Perú y Ecuador menos del 10\% y en personas con un nivel superior ya sea completa o incompleta en los países de Bolivia, Brasil, Colombia y Ecuador superan el $80 \%$ con acceso a una línea de internet.

Otro factor es la ubicación geográfica debido a que las personas de zonas urbanas tienen más acceso que los habitantes de zonas rurales; mientras que, el factor familia está representado por la cantidad de hijos en edad escolar que tienen mayor acceso por necesidad educativa, de los que no tienen hijos en edad escolar. Se ha notado también que existe la brecha de género, visible en algunos países más que en otros. En el caso de Bolivia, el 38\% de las mujeres usan internet y un $45 \%$ de los hombres, con una diferencia a notable de $7 \%$. En el Perú, se mantiene la diferencia del 7\%, en países de Uruguay, Colombia y Paraguay no se observa brecha de género, mientras que en Brasil el 59\% de las mujeres tienen acceso y el 58\% de los hombres, los niños y adultos mayores también tienen deficiencias en el acceso a internet.

Para superar las dificultades, la Unesco recomienda priorizar la educación con equipos tecnológicos digitales y líneas de internet de banda ancha para el logro de competencias, el desarrollo tecnológico del país y realizar la alfabetización digital a maestros y padres de familia. Esto se complementa también con brindar una línea de banda ancha para personas sin acceso a internet con el objetivo de mejorar la educación en el hogar y para que los adultos mayores tengan acceso a gestiones en línea, como el acceso a la salud y trámites administrativos. Esto implementando condiciones de inversión de subsidios a los hogares señalados para la asistencia escolar, apoyo a las personas con discapacidad. Todo ello es un reto para los países con condiciones de bajos ingresos económicos, pero aplicando políticas en función de necesidades de cambio, respetando el derecho a la educación con interculturalidad y cumpliendo con la meta de la inclusión social se estaría cerrando la brecha digital (Brito, 2017).

\section{Conclusiones}

Se concluye que la desigualdad en la educación digital está en proceso de cierre de brechas pues se observa que hay diferencias, distancia y separación en el acceso de herramientas tecnológicas, en el uso de herramientas digitales, competencias digitales y falta de acceso a conexión de línea de internet, para el desarrollo de sus experiencias de aprendizaje de los estudiantes. Esto último por causas como el factor económico, porque los jefes del hogar no están en posibilidades de adquirir herramientas tecnológicas digitales y mucho menos tener acceso a una línea de internet residencial, debido a que poseen otras prioridades básicas.

Por otro lado, tenemos que el nivel educativo como otro factor, debido a que las personas con mayor grado de instrucción son los que poseen herramientas digitales y acceso a líneas de internet. Ello, es visible en el análisis de resultados de países como como Bolivia, Brasil, Colombia, Ecuador, por citar algunos, se supera el $80 \%$ en el acceso digital e internet; mientras que, los países bajos como Honduras, Ecuador, Colombia 
y Bolivia, los que tienen nivel primario incompleta están entre 5\% a 10\% con acceso digital. Además, las familias con niños en el colegio también superan en el acceso de internet a las familias que no tienen hijos en el colegio. Ello nos lleva a mencionar que la necesidad de buscar un cambio y progreso en su familia se refleja en la necesidad de adquirir herramientas y acceso a internet para el logro de sus metas educativas.

Por otro lado, los que tienen la ubicación demográfica, es decir, estudiantes de zonas urbanas y rurales es más notorio, en contraste a las razones de ubicación demográfica donde la accesibilidad de líneas de internet y acceso a medios digitales es mínima por su excesivo costo. Adicionalmente, la brecha de género se diferencia por ser adulto mayor, una persona con discapacidad, entre otros. Se puede precisar que la brecha digital está asociada a la pobreza y que ha sido descubierta con más detalle a causa de esta pandemia del COVID-19. Esto puso en escenario una serie de posibilidades como la educación remota y que esta experiencia novedosa de la educación virtual debe ser tomada como una oportunidad. Donde el docente debe integrar las tecnologías de la información al proceso formativo de los estudiantes e incluir el apoyo de los padres de familia como guía en el hogar. Además, se requiere la disposición de los gobiernos, planteando sistemas y políticas educativas en beneficio de los estudiantes.

Se concluye que hay gobiernos, como el peruano, que tuvieron reacciones rápidas y adecuadas para la continuidad de la educación digital planteando estrategias educativas como: Aprendo en casa. Dicha estrategia contempló experiencias de aprendizaje por radio, televisión y plataforma web, para complementar el normal desarrollo de las clases los docentes. Y, se empezaron a utilizar plataformas educativas que incluyeron herramientas digitales para ser más efectivo. Viendo esa necesidad, el Ministerio de Educación del Perú, con el objetivo de cerrar la brecha digital, hizo entrega de herramientas electrónicas a los estudiantes y maestros de las zonas rurales considerados en el quintil uno y dos de pobreza. De esta manera, se logró una disminución de la brecha digital en la comunidad educativa, pudiéndose considerar que las herramientas tecnológicas, las habilidades del uso y la conexión a línea de internet son de vital importancia para el desarrollo de una educación de calidad con inclusión social e intercultural.

\section{Referencias}

Aguilar, S. M., y Chiang, M. M. (2020). Factores que determinan el uso de las TIC en adultos mayores de Chile. Revista Cientifica, 39(3), 296-308. https://doi.org/10.14483/23448350.16054

Barón, C. Y., y Caicedo, E. (2021). Transformación digital, un desafío en la educación superior. INVENTUM, 16(30). https://bit.ly/3x6KeXT

Bellver, V. (2019). Educar en Derechos Humanos: orientaciones del Derecho internacional e implementación en la educación superior. Revista de Educación y Derecho, (20). https://doi.org/10.1344/reyd2019.20.30022

Bonami, B., Piazentini, L., \& Dala, A. (2020). Education, Big Data and Artificial Intelligence: Mixed methods in digital platforms [Educación, Big Data e Inteligencia Artificial: métodos mixtos en plataformas digitales], Comunicar, 28(65), 43-52. https://doi.org/10.3916/C65-2020-04

Brito, L. (2017). Sociedad digital: brechas y retos para la inclusión digital en América Latina y el Caribe. Policy Papers Unesco.

Cisneros, V. (2017). Las Tecnologías de la Comunicación, ¿Incorporación/Exclusión Educativa en Guanajuato? Revista Iberoamericana para la Investigación y el Desarrollo Educativo, 7(14), 325-344. https://doi.org/10.23913/ride. v7i14.287

Congreso Latinoamericano de Telecomunicaciones. (2019). Cerrar la brecha digital en América Latina y el Caribe depende criticamente de la transformación de los Fondos de Servicio Universal | CAF. Banco de Desarrollo de América Latina.

Cortés, J. (2010). ¿Qué es la brecha digital?: una introducción al nuevo rostro de la desigualdad. Investigación Bibliotecológica: archivonomía, bibliotecología e información, 23(48). https://bit.ly/2TrSuDP 
Corzo, G. D., \& Alvarez, E. L. (2021). Technological competitiveness strategies in mobile connectivity and industry 4.0 communications in Latin America. [Estrategias de competitividad tecnológica en conectividad móvil y comunicaciones de la industria 4.0 en América Latina]. Información Tecnologica, 31(6), 183-192. https://bit. ly/3rp70c2

Cotino, L. (2021). La enseñanza digital en serio y el derecho a la educación en tiempos del coronavirus. Revista de Educación y Derecho, 21. https://doi.org/10.1344/reyd2020.21.31283

De Andrés, S., Collado, R., y García, J. I. (2020). Brechas digitales de género. Una revisión del concepto. Etic@net. Revista científica electrónica de Educación y Comunicación en la Sociedad del Conocimiento, 20(1). https://doi. org/10.30827/eticanet.v20i1.15521

Esteban, E. R., Cámara, A. A., y Villavicencio, M. del C. (2020). La educación virtual de posgrado en tiempos de COVID-19. Revista de Estilos de Aprendizajes, 13, 82-94. https://bit.ly/3xZpvWS

Gómez, Y. K. (2021). Estrategia Aprendo en Casa y la Competencia se comunica oralmente de los estudiantes de primaria. Episteme Koinonia, 4(7). https://doi.org/10.35381/e.k.v4i7.1172

Gómez, I., y Escobar, F. (2021). Educación Virtual en Tiempos de Pandemia: Incremento de la Desigualdad Social en el Perú. Chakiñan, Revista de Ciencias Sociales y Humanidades. https://chakinan.unach.edu.ec/index.php/ chakinan/article/view/553

Granado, M. (2019). Educación y exclusión digital: los falsos nativos digitales. Revista de estudios socioeducativos: RESED, 7. https://bit.ly/3kWp2kX

Hernández, R. y, y Mendoza, C. (2018). Metodología de la investigación Las rutas Cuantitativa, Cualitativa y Mixta. Editorial Mc Graw Hill Education. https://bit.ly/2Tqt1KX

Instituto Nacional de Estadística e Informática. (2018). Adultos mayores de 70 y más años de edad, que viven solos sobre la base de los resultados de los Censos Nacionales 2017: XII de Población, VII de Vivienda y III de Comunidades Indigenas, 1, 1-36.

Instituto Nacional de Estadística e Informática. (2019). Acceso de los hogares a las Tecnologías de Información y Comunicación (TIC). Instituto Nacional De Estadistica Informática, 4, 1-55.

Martínez, R. (2018). Evolución y tendencias de la formación a distancia y virtual. Investiga: Revista de Investigación en Gestión administrativa y Ciencias de la Información, 2. https://doi.org/10.23850/25907662.1851

Martínez, O. (2020). Brecha digital educativa. Cuando el territorio es importante. Sociedad e Infancias, 4, $267-270$. https://doi.org/10.5209/soci.69629

Menese, P. (2020). Desigualdad educativa en la Educación Media uruguaya. Páginas de Educación, 13(2), 34-58. https://bit.ly/3eIF6m8

Ministerio de Educación del Perú. (2020a). Resolución Ministerial 400-2020-minedu. https://bit.ly/3rnWPoc

Ministerio de Educación del Perú. (2020b). RVM Nº 093: Orientaciones Pedagógicas para el Servicio Educativo de Educación Básica durante el año 2020 En el Marco de la Emergencia Sanitaria por el Coronavirus Covid-19. El Peruano. https://bit.ly/3kHIOAl

Montenegro, S., Raya, E., y Navaridas, F. (2020). Percepciones Docentes sobre los Efectos de la Brecha Digital en la Educación Básica durante el Covid-19. Revista Internacional de Educacion para la Justicia Social, 9(3), 317-333. https://doi.org/10.15366/RIEJS2020.9.3.017

Presidencia del Consejo de Ministros. (2020). Decreto Supremo 044-2020. El Peruano, 90, 10-13. https://bit. ly/2W7AEah

Prince, Á. C. (2021). La brecha digital como obstáculo al derecho universal a la educación en tiempos de pandemia. Journal of the Academy, 4. https://doi.org/10.47058/joa4.3

Ruiz, A. (2017). Propuesta para la integración de TIC en la docencia de la linea curricular de formación integral de la Universidad San Sebastián (Sede Santiago). [Tesis de maestría, Pontificia Universitaria de Chile]. https:// repositorio.uc.cl/handle/11534/21451 
Sánchez, H. y Reyes, C. (2015). Metodología y Diseños en la Investigación Científica. Business Support.

Sandoval, C. H. (2020). La Educación en Tiempo del Covid-19 Herramientas TIC: El Nuevo Rol Docente en el Fortalecimiento del Proceso Enseńanza Aprendizaje de las Prácticas Educativa Innovadoras. Revista TecnológicaEducativa Docentes 2.0, 9(2). https://doi.org/10.37843/rted.v9i2.138

Toyama, J., y Rodríguez, A. (2019). Algoritmos laborales: big data e inteligencia artificial. THEMIS Revista de Derecho, 75. https://bit.ly/2V5fnO0

Valdez, J. (2018). Investigacion Cualitativa. Claves Teóricas y Prácticas. https://bit.ly/3iBqm9L

Velásquez, R. (2020). La Educación Virtual en tiempos de Covid-19. Revista Científica Internacional, 3(1), 19-25. https://bit.ly/3xXxh3L

Yansen, G., y Zukerfeld, M. (2013). Códigos generizados La exclusión de las mujeres del mundo del software, obra en cinco actos. Universitas Humanistica, 76(76), 207-233. https://bit.ly/2UBmcqr 